\title{
High symbol-rate polarization interference cancelation for satellite-to-ground remote sensing data transmission system
}

\author{
Zhisong $\mathrm{HaO}^{1,2^{*}}$ and Zhiming Zheng
}

\begin{abstract}
To remove the impacts of polarization interference on satellite-to-ground high-speed data transmission systems, the major factors that affect polarization interference are concluded, the deteriorations of bit error rate (BER) of different modulation systems and channel coding modes are simulated, and the parallel adaptive filtering technology is presented to resolve the high symbol-rate signal transmission polarization interference. It has been verified by experiments that the effects of this method is remarkable. In the situation of QPSK system, 500MSPS symbol rate and $10 \mathrm{~dB}$ polarization isolation, the BER performance can be improved $4.5 \mathrm{~dB}$ by using this method.
\end{abstract}

Keywords: Polarization interference, Polarization multiplexing, Satellite-to-ground data transmission system, BER

\section{Introduction}

In recent years, cognitive radio [1-3] and polarization multiplexing technology have become a research hotspot to improve the satellite remote sensing transmission system. The remote sensing satellites start to use polarization multiplexing technology to transmit data to improve the information transmission rate at home and board $[4,5]$. The polarization multiplexing technology uses the same spaceborne transmitting antenna and the same ground receiving antenna to simultaneously transmit the two carrier signals with the same frequency and the different polarization directions. When using the circularly polarized carrier, we select the left-hand polarized wave and the right-hand polarized wave to transmit information at the same time $[6,7]$.

Carrier polarity depends on the polarization characteristics of transmitting antenna feeder system, and the receiving antenna must have the same polarization with the transmitting antenna to implement polarization matching [8-10]. If the polarization is matched, all the energy can be received. If partially matched, only part of the energy can be received. If orthogonal, no energy can be received [11].

\footnotetext{
* Correspondence: 780025976@qq.com

${ }^{1}$ Mathematics and Systems Science Institute of Beihang University, Satellite Communication Technology, Math Information and Behavior Key Laboratory of MOE, Beijing 100191, China

${ }^{2}$ The 54th Research Institute of China Electronics Technology Group Corporation, Shijiazhuang 050081, China
}

For the reason of the antenna itself and the use of it, there has been polarization interference between the two polarization multiplexing signals $[12,13]$.

Using polarization multiplexing mode to transmit satellite-to-ground data, the problem of polarization interference must be solved. Polarization interference is mainly influenced by three aspects when the weather is fine $[14,15]$.

(1) Polarization isolation of on-satellite antenna

(2) Polarization isolation of ground receiving antenna

(3) Polarization deviation angle from satellite to ground

Theoretically, two orthogonal polarized waves are completely isolated, which means that an antenna can be configured with two receiving or sending ports, each of which matches only one polarized wave and is orthogonal to the other. In satellite communication systems, it is useful to use the characteristics of orthogonally polarized waves as additional isolation when transmitting and receiving in adjacent channels. However, due to the error of the actual transceiver equipment and the depolarization of the rain during the propagation of the radio wave, the polarization direction of the radio waves at the receiving end has generated errors, which will lead to two results: (1) the useful signals transmitted by the positive polarization mode will leak in the cross polarization direction and 
form interference to the cross polarization, and (2) the useful signals received in the direction of positive polarization will be weakened due to the error. The polarization isolation index does not meet the standard, which not only causes the transmission signal polarization attenuation in the positive polarization direction but also causes the leakage signal in the direction of reverse polarization to interfere the service on the frequency band, thereby affecting the quality of the receiving service.

The satellite-to-ground polarization deviation angle refers to the inclination of the antenna feed waveguide port relative to the ground caused by the difference between the position of the ground receiving base station and the satellite's location longitude, as well as the curvature of the earth. Therefore, the perfect deviation angle enables the receiving antenna to be well matched to the satellite polarization to receive weak satellite signals with high efficiency; otherwise, the signal may not be received.

The influence of these three aspects will make polarization match in the state of partial match [16]. Among them, polarization deviation angle from satellite to ground can be adjusted on the ground, so that its effect on data transmission system is much less than the effect of antenna isolation. That is to say, the main factor which effects data transmission system is the polarization isolation of antenna [17].

Based on investigation and research, the technical indicator of antenna polarization isolation of Ka-band carrier can be higher than $20 \mathrm{~dB}$. But in case of special weather, the polarization isolation can drop to $10 \mathrm{~dB}[18]$.

\section{Method}

The principle of the polarization interference suppression with adaptive filtering is as follows: the mutual interference degree of symbols in different polarization channels is constant in a certain period of time, the coefficients can be adjusted using minimum mean square error extraction and polarity correlation methods, and the system convergence can reach a stable value; after all of the symbols and coefficients are weighted, the polarization interference suppression is accomplished.

Adaptive filtering not only can suppress polarization interference but also can reduce the impacts of amplitude-frequency characteristics, phase-frequency characteristics, and orthogonal properties in channel on BER performance.

It is required to determine the structure, the error extraction algorithm, and the tap number of the digital adaptive filter. The limiting conditions are the accomplished hardware resources and processing speed.

\subsection{Adaptive filtering modes}

Based on the different implementation methods, the adaptive filtering modes of data transmission system can be divided into analog adaptive filtering and digital adaptive filtering. Analog adaptive filtering technology is to add an analog filter at the front end of demodulator to adapt channel amplitude and group delay characteristic. This technology cannot adjust with channel changes, has poor adaptability, and cannot solve orthogonal unbalance impact of microwave modulator. Digital adaptive filtering technology has strong adaptability, highly matches with channel, and can rectify orthogonal unbalance, thus its overall effect is superior to analog adaptive filtering technology. Therefore, digital adaptive filtering technology is adopted in high-speed data transmission system.

Digital adaptive filtering technology can be implemented either in frequency domain or in time domain. Frequency domain adaptive filter has poor compensation capability for group delay distortion, and it is commonly powerless especially for non-minimum phase fading. But time domain adaptive filter can adaptively filter both amplitude distortion and phase distortion at the same time, and it has the same adaptive filtering capability for both minimum phase fading and non-minimum phase fading. In addition, digital adaptive filter also has the characteristics such as easy to parallel and convenient to cascade clock carrier loop. Therefore, we adopt the digital time domain adaptive filter.

\subsection{Structure of adaptive filter}

The structure of time domain adaptive filter is linear transversal adaptive filter or decision feedback adaptive filter.

Transversal adaptive filter with linear structure has high feasibility, and has the same adaptive filtering capability for tailing interference and leading interference. While compensating for fading, it increases noise, and it has poor adaptive filtering capability for heavy fading. Compared with transversal adaptive filter, the characteristics of decision feedback adaptive filter include low tap number and lower operation throughput. If the decision is correct, each feed tap does not increase noise, and has better adaptive filtering capability for heavy fading.

In combination with the characteristics of satellite data transmission, the major factors of inter-symbol interference include microwave channel group delay and amplitude unbalance, modem orthogonal unbalance, power amplifier nonlinearity, and so on, which mainly cause signal inter-symbol tailing interference and cross interference. Satellite high-speed data transmission systems mostly adopt Ka band, which is little influenced by multipath factor, hence it will not generate channel heavy 


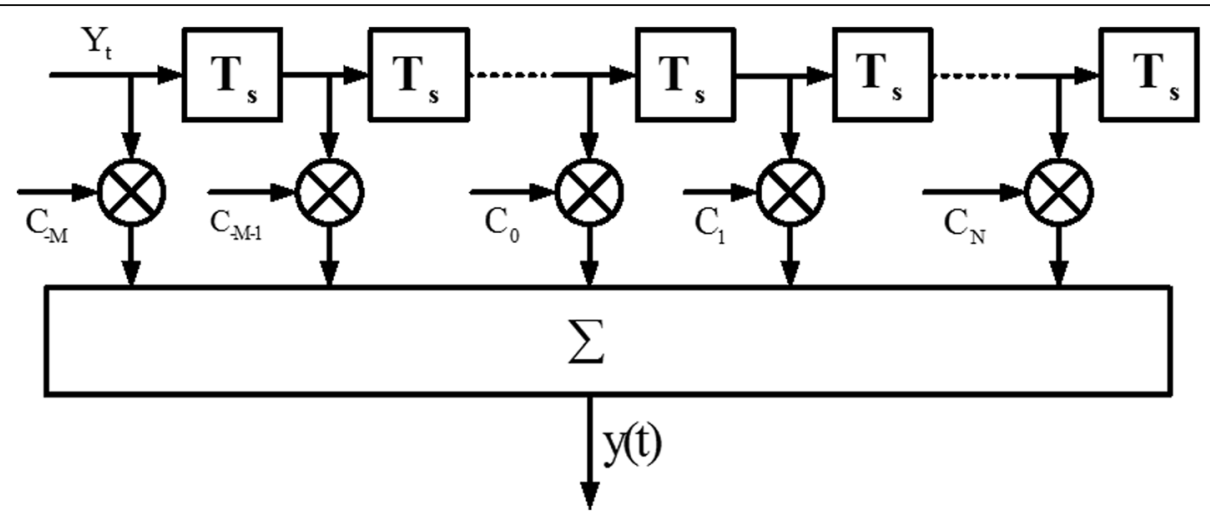

Fig. 1 Transverse linear adaptive filter structure. This figure shows a structure of transverse linear adaptive filter

fading in general. Therefore, the transversal linear structure can meet the system requirements.

In addition, it is required to adopt the pipeline structure in design of high-speed digital adaptive filtering circuit to solve time sequence problems. Adopting this structure, transversal linear adaptive filter is easy to implement. While decision feedback adaptive filter with high symbol rate, which implements timely adjusted coefficient, has many difficulties in data synchronization. Therefore, from the aspect of digital circuit implementation, the easily implemented transversal linear structure should be selected.

The transversal linear adaptive filter structure adopted by this paper is as shown in Fig. 1 .

\subsection{Error extraction algorithm}

Adaptive filter automatically adapts the change of channel characteristics by adjusting the gain of each tap to achieve the best filtering effect. At present, the conventional adaptive filtering error extraction algorithms include zero forcing algorithm, minimum mean square error algorithm, constant modulus algorithm, and so on. Zero forcing algorithm is applicable to the conditions that give priority to inter symbol crosstalk and in which noise is relatively minor, such as the wired channel. In wireless systems, the effect of adaptive filter using zero forcing algorithm is poor due to noise influence. Constant modulus algorithm uses the high order statistic information of modulus for channel adaptive filtering, and it has the characteristics of rapid convergence and minor steady state error. But its algorithm is relatively complicated, unsuitable for application in the high-speed data transmission systems. This paper adopts the minimum mean square error algorithm to adaptively update the tap coefficient of adaptive filter to track the channel changes.

The minimum mean square error algorithm can minimize the mean square error between the expected and actual output values of adaptive filter. It is widely used for characteristics such as less calculation and easy to implement. The minimum mean square error algorithm is based on the steepest descent principle, that is, search along the negative gradient direction of weight and achieve the optimal weight to minimize the filtered mean square error.

Assume that the input of filter is:

$$
U=[u(n), u(n-1), \Lambda u(n-M+1)]
$$

$M$ is the number of taps. The tap weight is:

$$
W=\left[\hat{w}_{0}(n), \hat{w}_{1}(n), \Lambda, \hat{w}_{M-1}(n)\right]
$$

The error is:

$$
e(n)=d(n)-y(n)=d(n)-W^{H}(n) U(n)
$$

LMS algorithm can be described as:

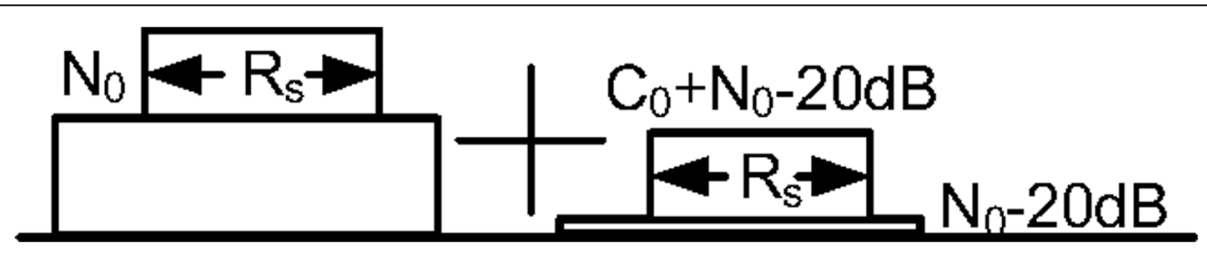

Fig. 2 Equal bandwidth polarization interference schematic diagram. This figure shows an equal bandwidth polarization interference schematic diagram 


$$
W(n+1)=W(n)+\mu e(n) U(n)
$$

Among the rest, $\mu$ is the step parameter used to control stability and convergence rate.

\subsection{Determination of tap number}

According to the satellite channel deterioration conditions and the simulation architecture, the tap number of adaptive filtering is determined as 33 . In addition to central tap, there are 2 forward taps and 30 backward taps.

\section{System model}

It is assumed that the signal transmission bandwidth is Nyquist bandwidth W, namely the forming coefficient is 0 ; here, the interference of the two signals that are in orthogonal polarization is as shown in Fig. 2.

Assume that symbol rate is Rs, and the following formula is workable:

$$
R_{s}=W ;
$$

Assume that information rate is $R_{b i t}, R_{b i t} / R_{s}=a$, and $a$ is related to modulation mode and coding efficiency.

$E_{b}$ is the energy per information bit. $C_{0}^{\prime}$ is power spectral density, and its logarithmic form is $C_{0} . N_{O}^{\prime}$ is noise power spectral density, and its logarithmic form is $N_{0}$.

Here:

$$
C_{0}^{\prime}=E_{b}^{\prime} R_{b i t} / R_{s}=E_{b}^{\prime} a ;
$$

Introduce polarization interference in this case. Assume that polarization isolation is $20 \mathrm{~dB}$. At this time, the ratio of energy per information bit to noise power spectral density is:

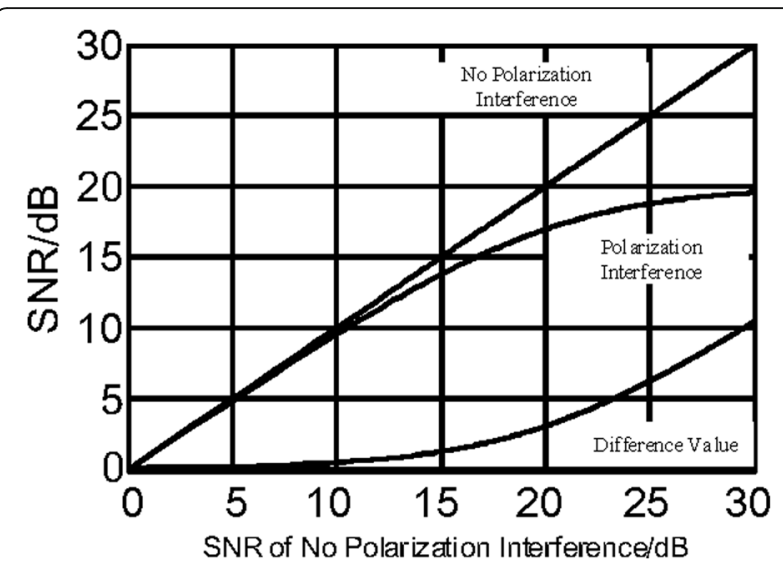

Fig. 3 Deterioration of $E_{b} / N_{0}$ in case of QPSK and channel coding. This figure shows a graph of deterioration of $E_{b} / N_{0}$ in case of QPSK and channel coding, $a=1$

$$
E_{b} / N_{0}-10 \lg \left[1+0.01\left(a E_{b}^{\prime} / N_{0}^{\prime}+1\right)\right]
$$

Therefore, the deterioration of $E_{\mathrm{b}} / N_{0}$ is:

$$
10 \lg \left[1+0.01\left(a E_{b}^{\prime} / N_{0}^{\prime}+1\right)\right] \text {. }
$$

The following is the analysis of several transmission signal systems:

\section{(1) QPSK+ $1 / 2 \mathrm{LDPC}$}

In this case, $a=1$, the deterioration of $E_{b} / N_{0}$ is as shown in Fig. 3.

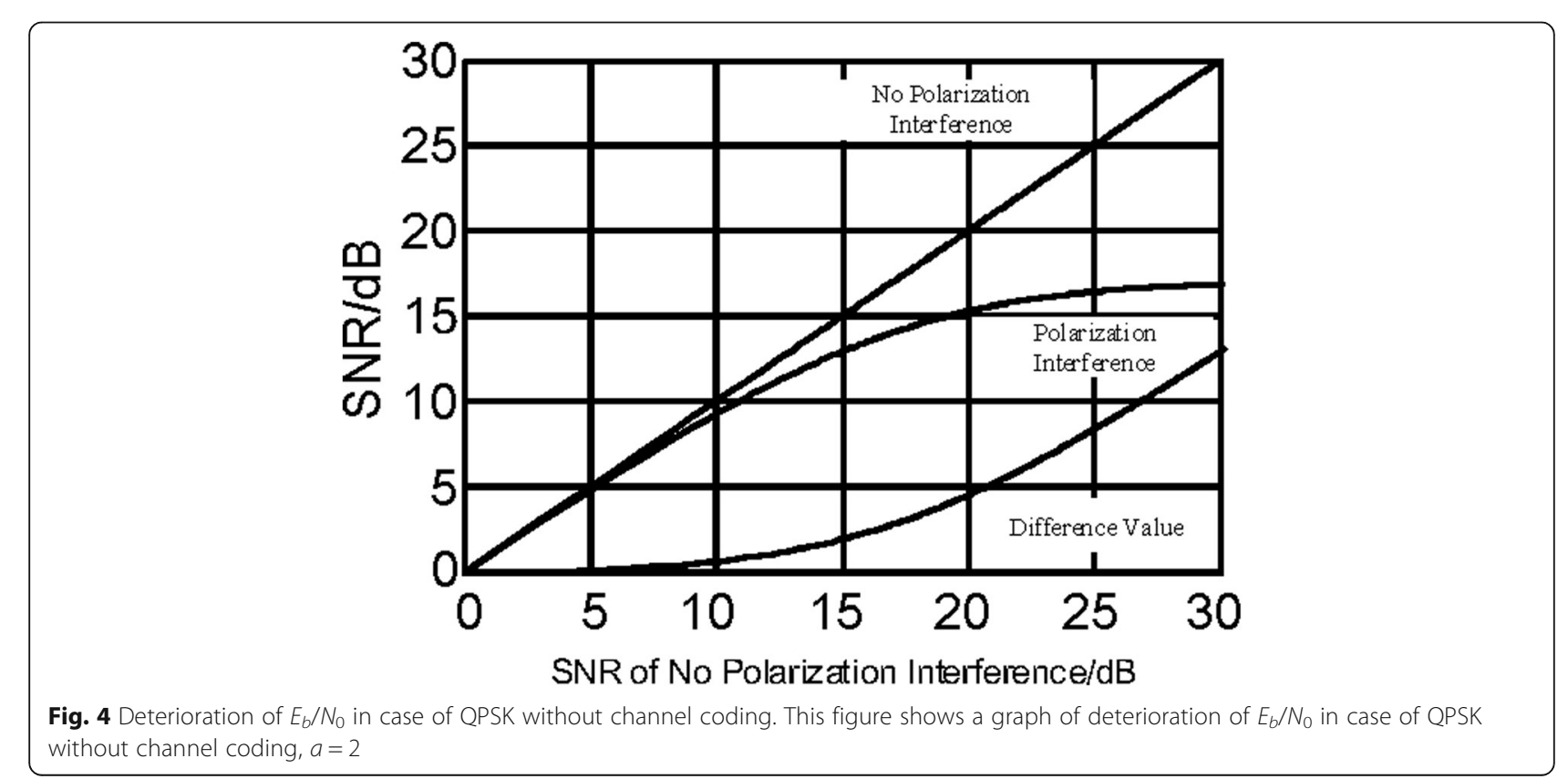




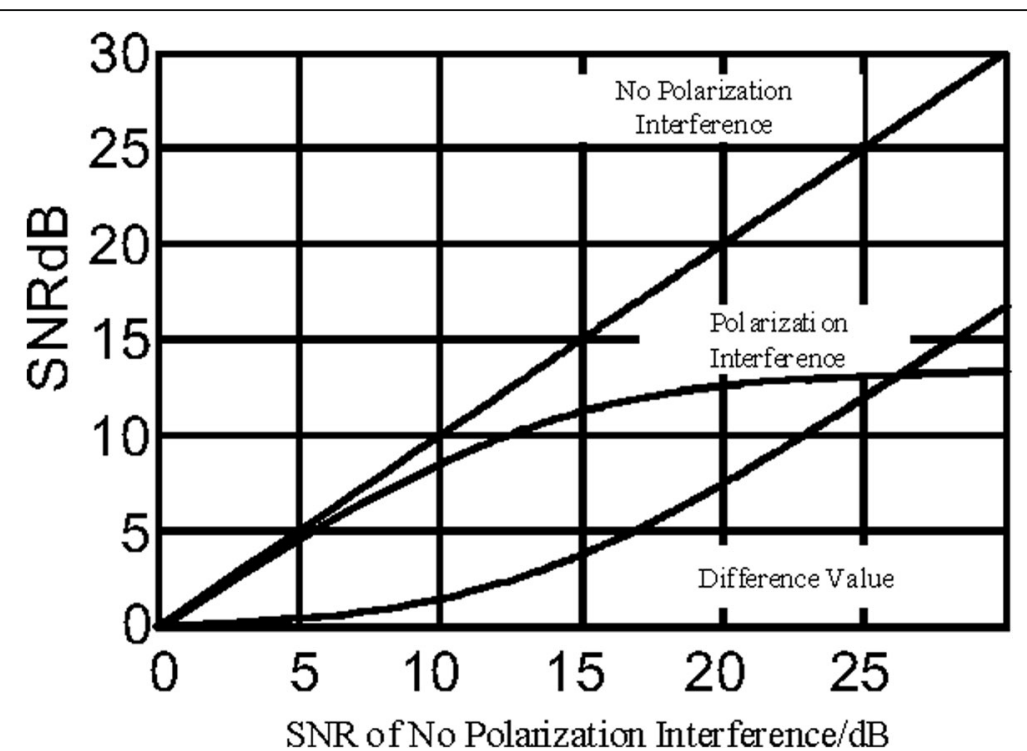

Fig. 5 Deterioration of $E_{b} / N_{0}$ in case of $16 \mathrm{QAM}$ without channel coding. This figure shows a graph of deterioration of $E_{b} / N_{0}$ in case of $16 \mathrm{QAM}$ without channel coding, $a=4$

For a demodulator with $2 \mathrm{~dB}$ demodulation loss, when $E_{b} / N_{0}$ is higher than $10 \mathrm{~dB}$, after polarization interference is introduced, $E_{b} / N_{0}$ is still higher than $9.5 \mathrm{~dB}$, and BER is less than $1 \times 10^{-8}$.

After polarization interference is introduced, deterioration of $E_{b} / N_{0}$ is at most $0.5 \mathrm{~dB}$ when $E_{b} / N_{0}$ is less than $10 \mathrm{~dB}$, and there is almost no deterioration when $E_{b} / N_{0}$ is less than $8 \mathrm{~dB}$, and it has little effect on BER.

In this case, the introduction of polarization interference has no effect on performance of data transmission system.

(2) QPSK without channel coding and decoding

In this case, $a=2$, the deterioration of $E_{b} / N_{0}$ is as shown in Fig. 4.

For a demodulator with $2 \mathrm{~dB}$ demodulation loss, when $E_{b} / N_{0}$ is higher than $20 \mathrm{~dB}$, after polarization interference is introduced, $E_{b} / N_{0}$ is higher than $15 \mathrm{~dB}$, and BER is less than $1 \times 10^{-9}$, which has little effect on system performance.

When $E_{b} / N_{0}$ is $15 \mathrm{~dB}$, after polarization interference is introduced, $E_{b} / N_{0}$ is $13 \mathrm{~dB}$, and BER increases from $1 \times$ $10^{-9}$ magnitude to $1 \times 10^{-8}$ magnitude.

When $E_{b} / N_{0}$ is $13 \mathrm{~dB}$, after polarization interference is introduced, $E_{b} / N_{0}$ is $11.5 \mathrm{~dB}$, and BER increases from $1 \times 10^{-8}$ magnitude to $1 \times 10^{-6}$ magnitude, which has some effect on system performance.

Because it is required that the BER of remote sensing satellite is generally less than $1 \times 10^{-7}$ and there remains certain power margin, the polarization interference has limited effect on system when this modulation mode is adopted.

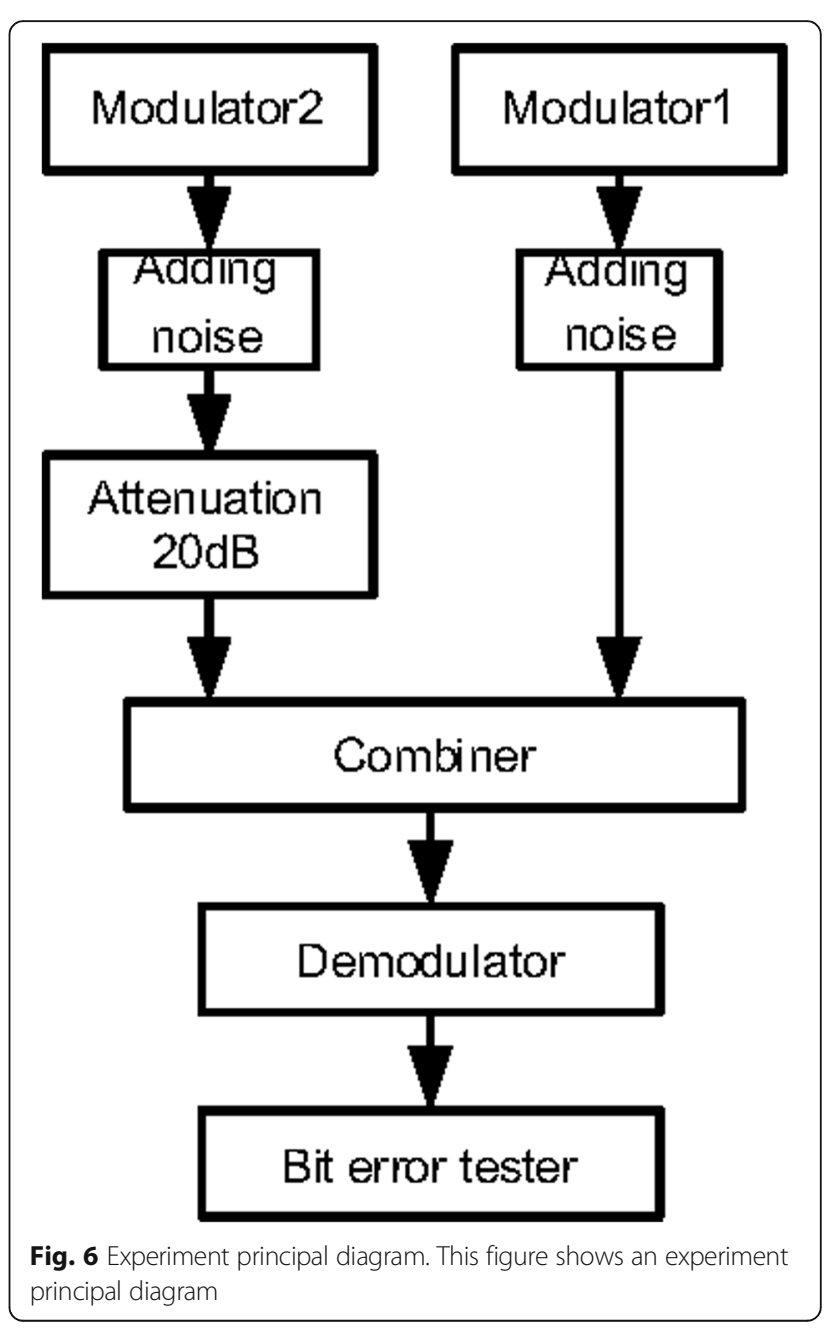




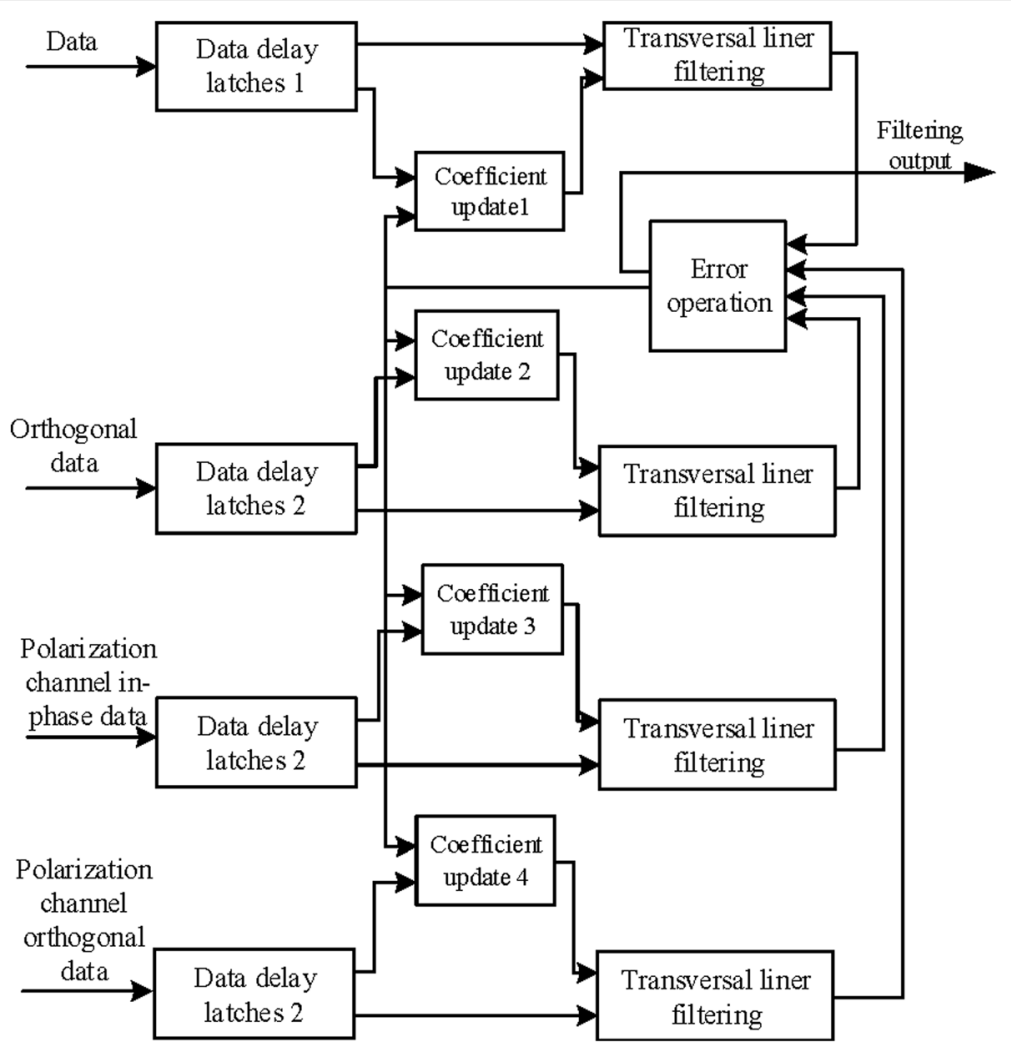

Fig. 7 Transversal linear structure after orhogonal improvement. This figure shows a transversal linear structure after orthogonal improvement

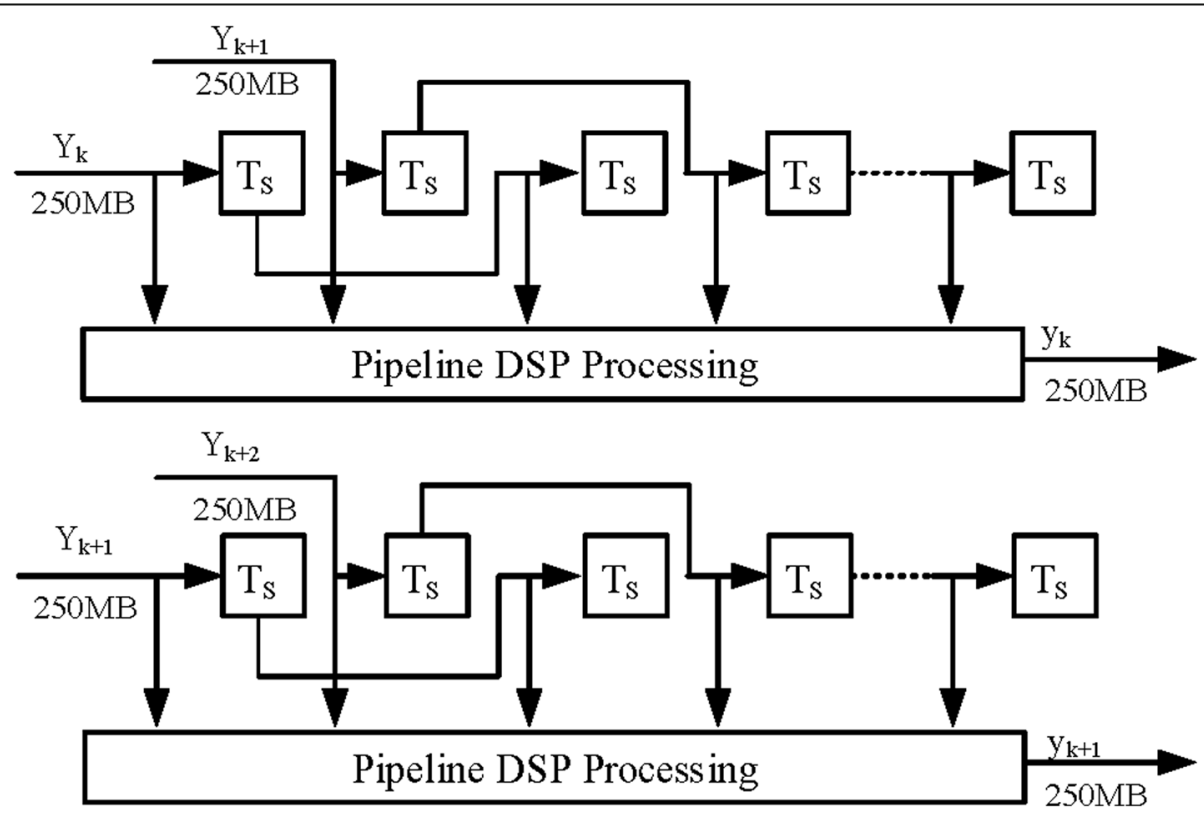

Fig. 8 Improved parallel structure. This figure shows an improved parallel structure 


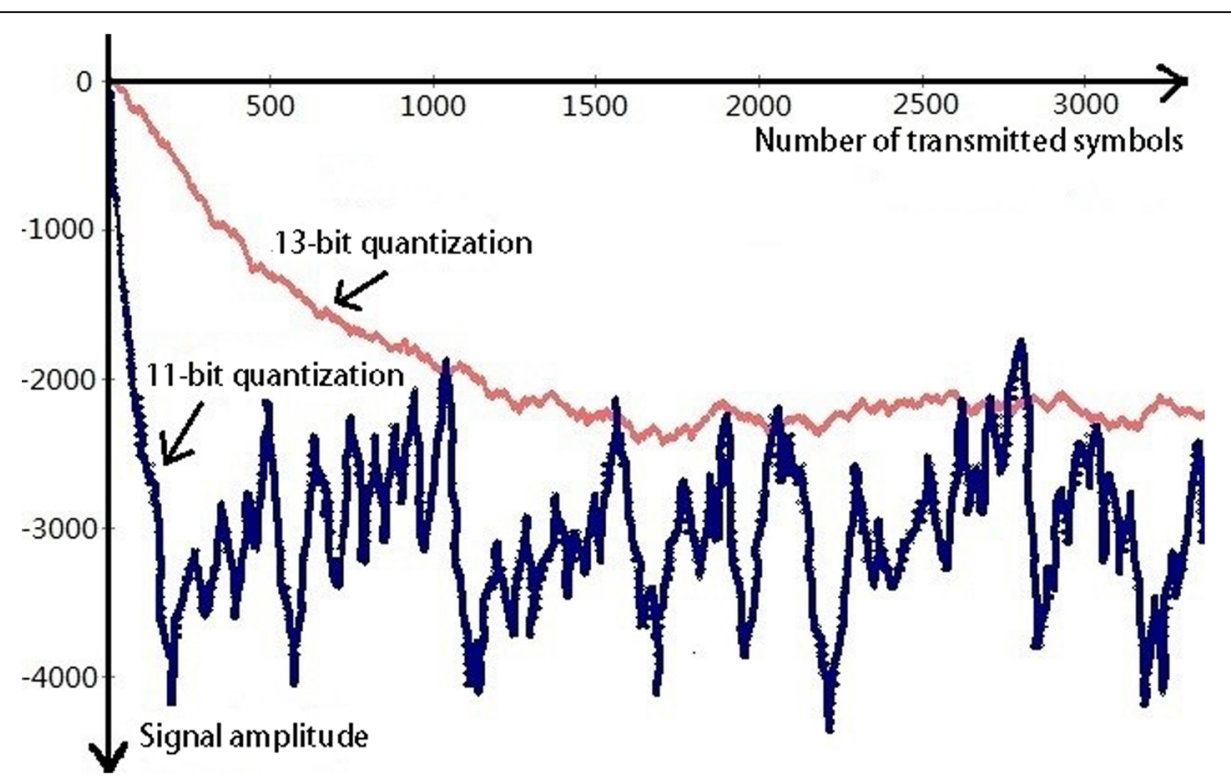

Fig. 9 Convergence and steady state errors of adaptive filter. This figure shows a graph of convergence and steady state errors of adaptive filter

(3) 16QAM without channel coding

In this case, $a=4$, the deterioration of $E_{b} / N_{0}$ is as shown in Fig. 5.

For a demodulator with $2 \mathrm{~dB}$ demodulation loss, when $E_{b} / N_{0}$ is $17 \mathrm{~dB}$, after polarization interference is introduced, $E_{b} / N_{0}$ is $12 \mathrm{~dB}$, and BER increases from $10^{-8}$ magnitude to $10^{-3}$ magnitude.

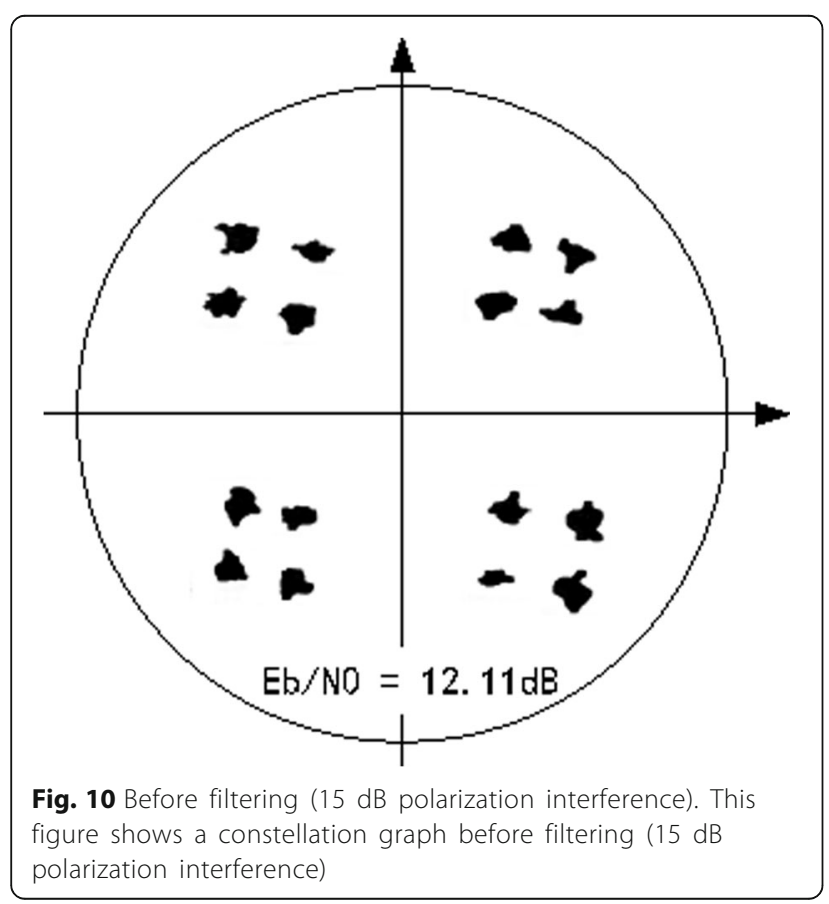

Adopting 16QAM modulation mode, in the case of no channel coding, it has great effect on demodulation performance and even make the system unable to work.

\section{Experiment}

4.1 Verification deterioration of polarization interference with experiment

We verify the polarization interference cases with experiments using the available hardware conditions to set up

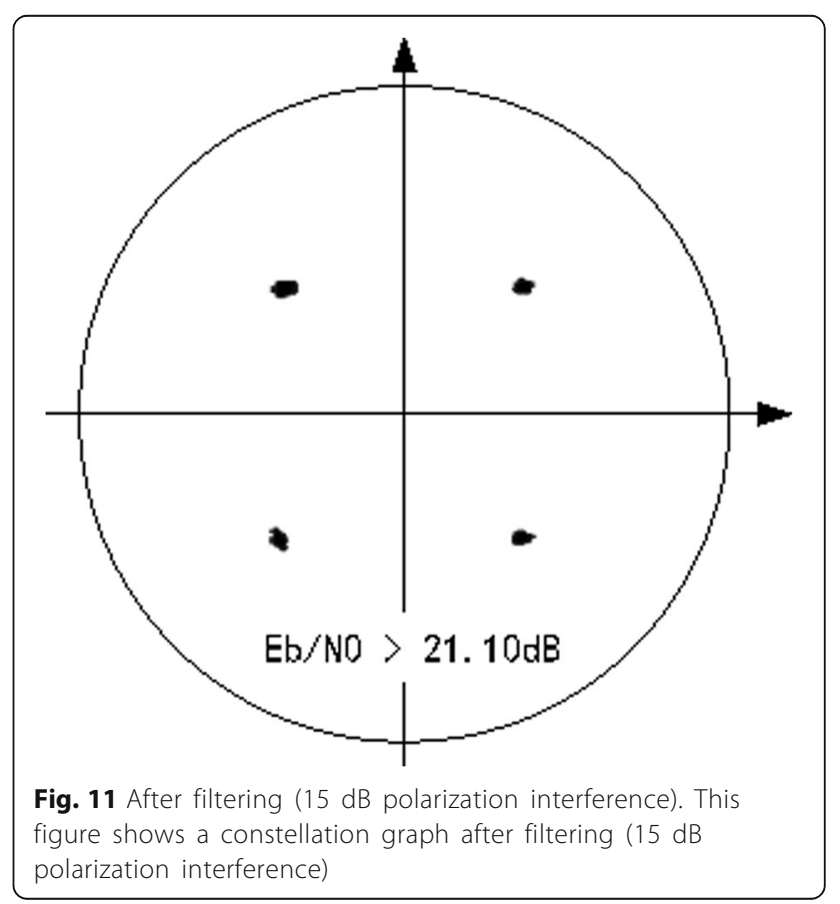




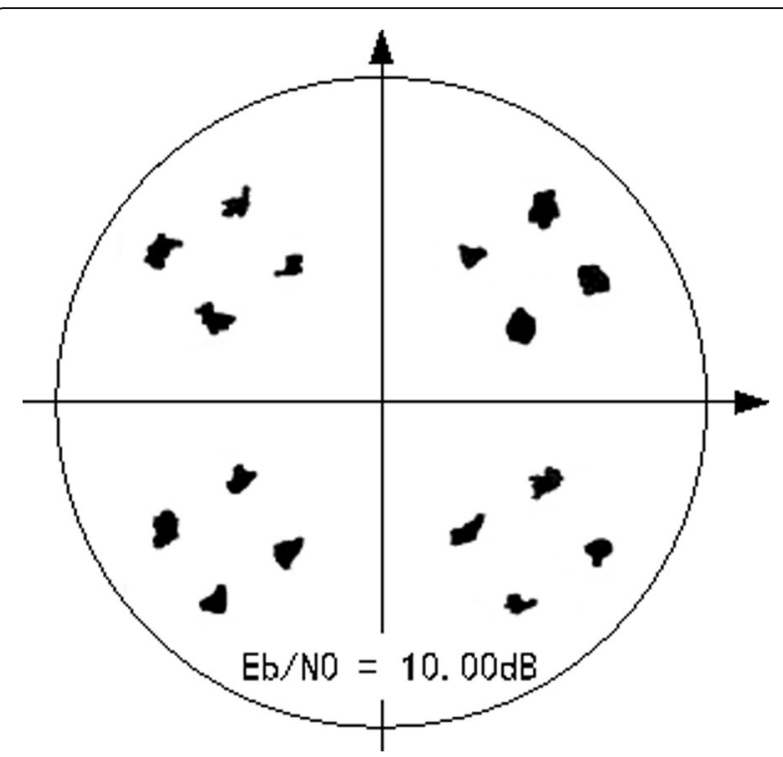

Fig. 12 Before filtering (10 dB polarization interference). This figure shows a constellation graph before filtering (10 dB polarization interference)

the test system. The symbol rate of test system is 500 Mbps. The principle diagram is as shown in Fig. 6.

We use the same two modulators, make them transmitting the same power, add the same white noise to them, and adjust the noise level of both to make the BER of system is less than $1 \times 10^{-7}$. One way of IF signal of which modulator is added noise is attenuated $20 \mathrm{~dB}$, and then is combined with the other way to access to

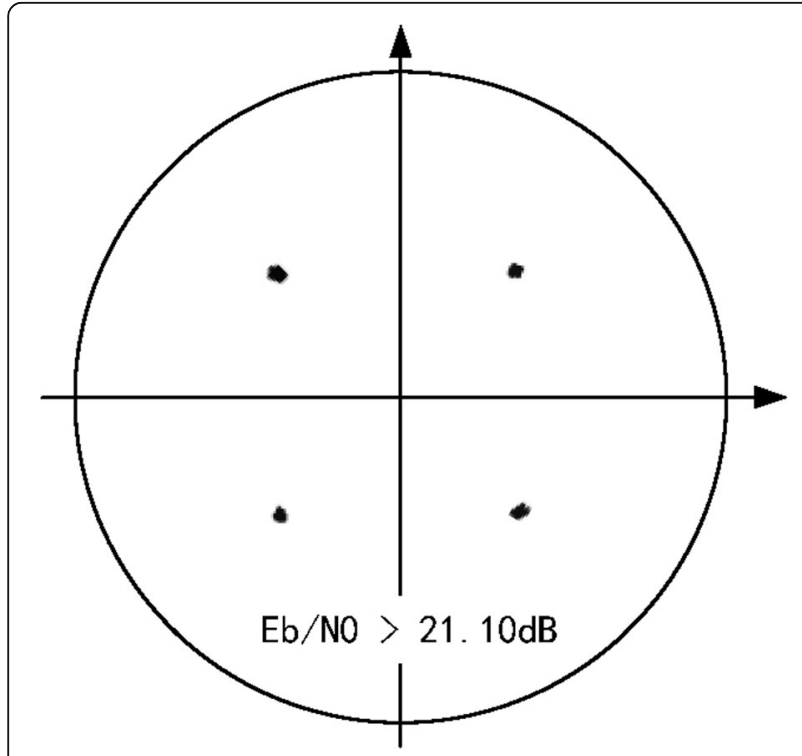

Fig. 13 After filtering (10 dB polarization interference). This figure shows a constellation graph after filtering $(10 \mathrm{~dB}$ polarization interference) the demodulator. Observe the changes of BER. The test results are as follows:

(1) Adopting QPSK system and LDPC coding of which coding efficiency is $1 / 2$; it has been tested that, before polarization interference, when $E_{b} / N_{0}$ is $4.35 \mathrm{~dB}, \mathrm{BER}$ is $2.35 \times 10^{-8}$, after polarization interference, $E_{b} / N_{0}$ is deteriorated to $4.25 \mathrm{~dB}$ and BER is $3.74 \times 10^{-8}$.

(2) Adopting QPSK system and no channel coding; it has been tested that, before polarization interference, when $E_{b} / N_{0}$ is $13.12 \mathrm{~dB}, \mathrm{BER}$ is $9.40 \times 10^{-8}$, after polarization interference, $E_{b} / N_{0}$ is deteriorated to $11.61 \mathrm{~dB}$, and BER is $3.22 \times 10^{-6}$.

(3) Adopting 16QAM system and no channel coding; it has been tested that, before polarization interference, when $E_{b} / N_{0}$ is $17.40 \mathrm{~dB}, \mathrm{BER}$ is $8.92 \times 10^{-8}$, after polarization interference, $E_{b} / N_{0}$ is deteriorated to $12.18 \mathrm{~dB}$, and BER is $2.67 \times 10^{-4}$.

The test results are basically consistent with the theoretical simulation analysis.

\subsection{Implementation of high-speed adaptive filter}

After the overall scheme of adaptive filter is determined, it is required to adopt the specific implementation method in accordance with the characteristics of high-speed data transmission system.

\subsubsection{Structure improvement}

In order to reduce the influences of polarization mutual interference and orthogonal unbalance, orthogonalization improvement is required for transversal linear adaptive filter. The data entering adaptive filter is not only used for filtering of its own branch but also used for filtering of the corresponding orthogonal branch and polarization branch. The one-way improved adaptive filter structure is shown in Fig. 7.

The coefficients of in-phase channel and orthogonal channel both are adjusted in accordance with the results of error budget. The result of in-phase data adaptive filtering minus the result of orthogonal data filtering leaves a result, and then this result is compared with the expected value to get the adaptive filtering error. The improved adaptive filter can decrease the inter symbol crosstalk that comes from the orthogonal channel.

\subsubsection{Parallel structure improvement}

Due to the restriction of digital devices, the transversal linear adaptive filter structure can achieve the highest $250 \mathrm{M}$ baud in FPGA, thus we must adopt the mode of two-way parallel processing. If the data are divided into two ways, each way is adaptively filtered separately, thus the processing speed of each way declines to the half of 


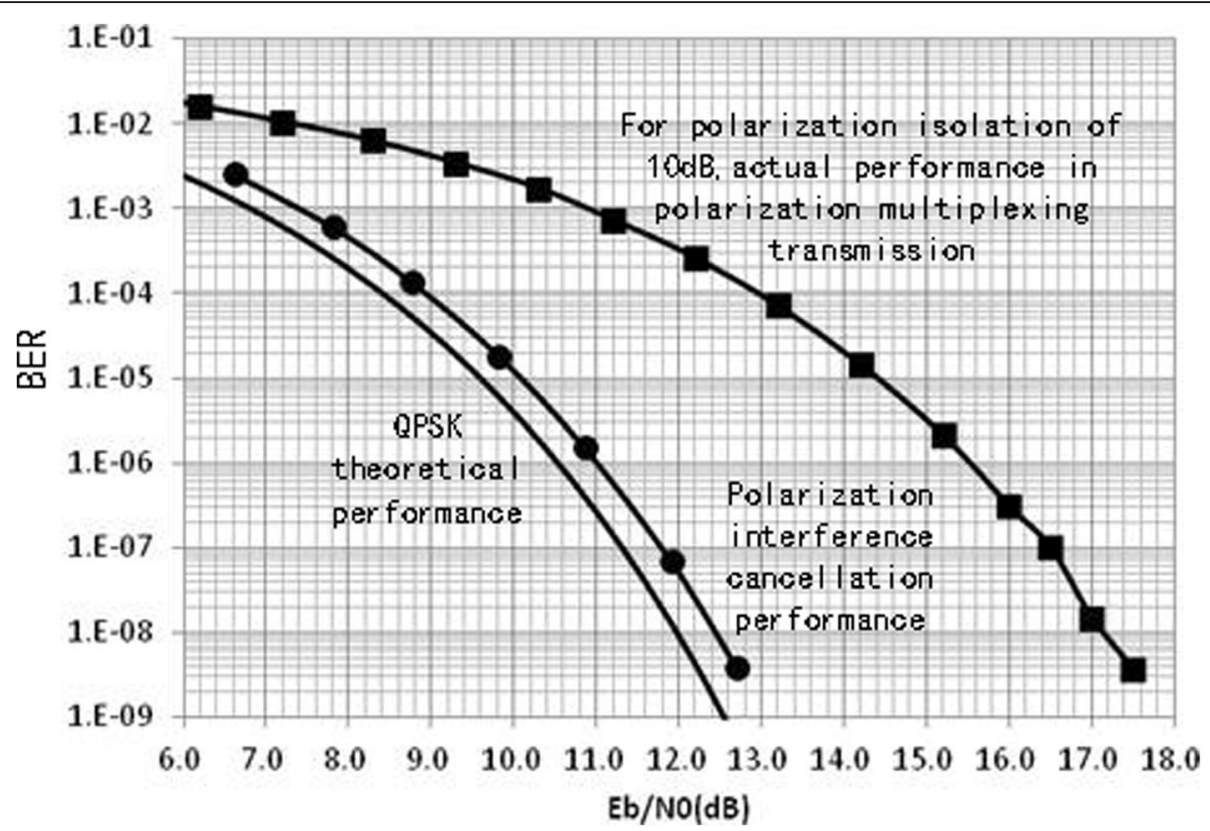

Fig. 14 Test situations before and after QPSK signal filtering. This figure shows a graph of test situations before and after QPSK signal filtering

serial processing speed, which seriously influences the effect of adaptive filtering. This is because the crosstalk generated by all of the symbols adjacent to the central tap and the symbols away from the central tap odd intervals cannot be adaptively filtered. And these effects are often very serious. In order to not only decrease the speed but also not influence the effect of adaptive filtering, we adopt the following improved parallel structure:

It can be seen from Fig. 8 that the results of each way signal processing of improved adaptive filter are dependent with all of the serial data input, and the processing speed of each way declines to the half of serial processing speed.

\subsubsection{Steady-state error decrease}

The pipeline structure is adopted in the coefficient adjustment circuit, and the error adjustment results are transmitted to the coefficient adjustment module in about ten cycles. The greater the coefficient adjustment delay, the bigger the steady state error. To solve this problem, it is required to increase the coefficient quantization accuracy and decrease the adjustment step. Thus the coefficient convergence time would increase. But the convergence symbolic number restriction can be relaxed properly in high-speed data transmission system. On balance, we select the coefficient quantization bit as 13 and adjustment step as 1 . The coefficient convergence and steady-state regulation conditions are shown in Fig. 9.

The above figure shows the convergence and steadystate error conditions of different coefficient quantization bit of adaptive filter. The blue line shows the convergence conditions of 11 bit quantization, and the red line shows the convergence conditions of 13 bit quantization. Changing the quantization bit corresponds to adjusting step. After the step is adjusted, the convergence time increases, but the steady-state error decreases.

\section{Simulation results}

It has been tested that the effect of polarization interference suppression is obvious at $500 \mathrm{M}$ baud symbol rate.

In results, the equivalent $E_{b} / N_{0}$ value is used to characterize the deteriorations of constellation points after polarization interference. The equivalent $E_{b} / N_{0}$ value is to convert the dispersion degree of constellation points to the corresponding energy per information bit and the equivalent noise power spectral density ratio.

In the circumstance of $15 \mathrm{~dB}$ polarization interference, the demodulated equivalent $E_{b} / N_{0}$ value of constellation points increases from $12.11 \mathrm{~dB}$ to above $21.10 \mathrm{~dB}$ through adaptive filtering, The results are shown in Figs. 10 and 11.

In the circumstance of $10 \mathrm{~dB}$ polarization interference, the demodulated equivalent $E_{b} / N_{0}$ value of constellation points increases from $10.00 \mathrm{~dB}$ to above $21.10 \mathrm{~dB}$ through adaptive filtering, The results are shown in Figs. 12 and 13.

In the circumstance of $10 \mathrm{~dB}$ polarization interference, the BER curves before and after filtering are shown as below.

It can be seen from Fig. 14 that the $E_{b} / N_{0}$ value after adaptive filtering differs by $0.5 \mathrm{~dB}$ from the theoretical 
value at 10-7 BER, improving $4.5 \mathrm{~dB}$ relative to without adaptive filter.

By the theoretical derivation, system simulation, and experimental verification, we conclude that as $a$ increases, $E_{b} / N_{0}$ working threshold increases gradually, and the effect of polarization interference on system BER performance also increases, in polarization multiplexing system with $20 \mathrm{~dB}$ isolation. When $E_{b} / N_{0}$ working threshold is less than $10 \mathrm{~dB}$, the system is almost unaffected; when $E_{b} / N_{0}$ working threshold is $10 \sim 15 \mathrm{~dB}$, the system BER performance decreases 1 2 order of magnitude. When $E_{b} / N_{0}$ working threshold is above $15 \mathrm{~dB}$, the system BER performance decreases seriously.

For isolation of $10 \mathrm{~dB}$, the general data transmission systems would be affected by polarization interference. QPSK system without coding and decoding would be affected seriously.

The polarization interference canceling technology based on adaptive filtering should be used at the receiving end to decrease the effect of polarization interference to a large extent and improve the performance of data transmission system.

\section{Conclusion}

Application of polarization multiplexing technology to the satellite-to-ground remote sensing data transmission can increase the bandwidth availability by decreasing the data transmission time. But if the polarization interference problem is not solved well, the transmission performance would decrease so much as breaking down the whole system. In charge of the rules of effect of polarization interference on satellite-to-ground data transmission system, decreasing the effect with digital adaptive filtering has reference significance in the construction of satellite-toground remote sensing data transmission system.

\section{Abbreviations}

BER: Bit error rate; FPGA: Field programmable gate array; LDPC: Low-density parity-check code; PSK: Phase shift keying; LMS: Least mean square

\section{Acknowledgements}

The authors would like to thank the reviewers for their thorough reviews and helpful suggestions.

\section{Funding}

This work was supported by the National Natural Science Foundation of China (No. 61402030).

\section{Availability of data and materials}

Data sharing not applicable to this article as no datasets were generated or analyzed during the current study.

\section{Authors' contributions}

$\mathrm{ZH}$ wrote the majority of the text and performed the design and implementation of the algorithm. ZZ contributed text to earlier versions of the manuscript and commented on and approved the manuscript. Both authors read and approved the final manuscript.

\section{Competing interests}

The authors declare that they have no competing interests.

\section{Publisher's Note}

Springer Nature remains neutral with regard to jurisdictional claims in published maps and institutional affiliations.

Received: 11 September 2018 Accepted: 21 November 2018

Published online: 12 December 2018

\section{References}

1. M. Jia, X. Gu, Q. Guo, et al., Broadband hybrid satellite-terrestrial communication systems based on cognitive radio toward 5G. IEEE Wirel. Commun. 23(6), 96-106 (2016)

2. M. Jia, X. Liu, X. Gu, et al., Joint cooperative spectrum sensing and channel selection optimization for satellite communication systems based on cognitive radio. Int. J. Satell. Commun. Netw. 35(2), 139-150 (2017)

3. M. Jia, X. Liu, Z. Yin, et al., Joint cooperative spectrum sensing and spectrum opportunity for satellite cluster communication networks. Ad. Hoc. Netw. 58, 231-238 (2017)

4. N. Zhao, Application of dual-polarized technology in remote sensing satellite data transmission. Spacecraft Eng 19(4), 55-62 (2010)

5. P.D. Karabinis, Atc Technologies L, Satellite communications systems and methods using diverse polarizations. Atc Technologies (2009)

6. V. Popovskyy, A.W.S.A. Iskandar, Polarization multiplexing modulation in fiber-optic communication lines. IEEE Problems of Infocommunications Science and Technology (2017), pp. 214-216

7. C.W. Hsu, C.H. Yeh, C.W. Chow, Using adaptive equalization and polarization-multiplexing technology for gigabit-per-second phosphorLED wireless visible light communication. Opt. Laser Technol 104, 206209 (2018)

8. J. Shapira, D. Levy, S. Miller, System and method for improving polarization matching on a cellular communication forward link: US, US7113748[P] (2006)

9. F.M. Yang, Analysis of polarization matching on satellite TV receiving antenna. China Cable Telev 2004(24), 69-72 (2004)

10. J. Zhang, Y. Huang, Z. Li, Analysis of polarization matching and transmitting/ receiving pattern reciprocity of antenna. IEEE Antennas and propagation (2014), pp. 248-251

11. Y. Kurokami, Cross polarization interference canceller and method of canceling cross polarization interference: U.S. Patent 7,016,438 (2006)

12. M.T. Core, Cross polarization interference cancellation for fiber optic systems. J. Lightwave Technol. 24(1), 305-312 (2006)

13. K. Kuhlmann, D. Jalas, A.F. Jacob, Mutual in Ka-band antenna array with polarization multiplexing. IEEE Wireless Technology Conference (2009), pp. 152-155

14. R.W. Kreutel, D.F. Difonzo, W.J. English, et al., Antenna technology for frequency reuse satellite communications. Proc. IEEE 65(3), 370-378 (1977)

15. Y. Sturkovich, E. Tsur, Communication system having cross polarization interference cancellation (XPIC) (2015)

16. D.P. Stapor, Optimal receive antenna polarization in the presence of interference and noise. IEEE Trans. Antennas Propag. 43(5), 473-477 (1995)

17. M. Zhang, L. Zhang, J. Wu, et al., Detection and compensation of basis deviation in satellite-to-ground quantum communications. Opt. Express 22(8), 9871-9886 (2014)

18. L. Castanet, A. Bolea-Alamañac, M. Bousquet, in Proc. 2nd International Workshop of COST Action. Interference and fade mitigation techniques for Ka and Q/N band satellite communication systems (2003), p. 280

\section{Submit your manuscript to a SpringerOpen ${ }^{\circ}$ journal and benefit from:}

- Convenient online submission

- Rigorous peer review

- Open access: articles freely available online

High visibility within the field

- Retaining the copyright to your article

Submit your next manuscript at $>$ springeropen.com 